\title{
Fullerene-Related Nanocarbons and Their Applications
}

\author{
Junfeng Geng, ${ }^{1,2}$ Kun'ichi Miyazawa, ${ }^{3}$ Zheng $\mathrm{Hu}^{4}{ }^{4}$ \\ Ilia A. Solov'yov, ${ }^{5}$ and Angel Berenguer-Murcia ${ }^{6}$ \\ ${ }^{1}$ Institute for Materials Research and Innovation, University of Bolton, Bolton BL3 5AB, UK \\ ${ }^{2}$ Department of Chemistry, University of Cambridge, Cambridge CB2 1EW, UK \\ ${ }^{3}$ Fullerene Engineering Group, Materials Processing Unit, National Institute for Materials Science, 1-1 Namiki, Tsukuba, \\ Ibaraki 305-0044, Japan \\ ${ }^{4}$ Key Laboratory of Mesoscopic Chemistry, School of Chemistry and Chemical Engineering, Nanjing University, Nanjing 210093, China \\ ${ }^{5}$ Beckman Institute for Advanced Science and Technology, University of Illinois at Urbana-Champaign, 405 N. Mathews Avenue, \\ Urbana 61801, USA \\ ${ }^{6}$ Materials Institute, University of Alicante, San Vicente del Raspeig, 03080 Alicante, Spain
}

Correspondence should be addressed to Junfeng Geng, j.geng@bolton.ac.uk

Received 24 July 2012; Accepted 24 July 2012

Copyright (C) 2012 Junfeng Geng et al. This is an open access article distributed under the Creative Commons Attribution License, which permits unrestricted use, distribution, and reproduction in any medium, provided the original work is properly cited.

The discovery of fullerene (C60) in 1985 spurred on the subsequent discoveries of a number of fullerene-related novel carbons at the nanometre scale. These nanocarbons are related to one another in structure, providing an interesting spectrum of variants which display an array of unique properties. From the vast amount of research that has been conducted over the last two decades, it is now apparent that these nanomaterials, notably, carbon nanotubes, carbon-based nanoparticles, graphene, fullerene and fullerene derivatives promise very distinct applications and will add great value to industries. It is thus expected that the studies on these nanocarbons and related technologies will have huge impacts on future nanotechnology and will significantly contribute to our economy and society.

The research in the field is at the crossroads between different technologies and disciplines involving materials science, chemistry, physics, engineering, and nanotechnology. Its implementation will greatly benefit new, high-tech industries and also help the transformation of traditional carbonbased industries from a resource-intensive to a knowledgeintensive base. However, major challenges exist in the area, which are mainly associated with providing answers to key questions such as how to control the nanostructures, how to produce them in commercial quantities but at relatively low costs, and how to apply them by specific requirements. In this special issue on fullerene-related nanocarbons and their applications, contributions from a broad field of research have been received from worldwide scientists. The published works are briefly outlined as follows.

The paper by C. H. Lin et al. is a report on the effects of doping graphene in poly(3,4-ethylenedioxythiophene): poly(styrene sulfonate), PEDOT:PSS, as a PEDOT: PSS/graphene nanocomposite hole injection layer on the performance enhancement of polymer light-emitting diodes. The devices are fabricated with the nanocomposite layer, and the influence of the doping concentration on the device performance is examined by measuring the device output properties. Another paper by N. A. D. Yamamoto et al. addresses a similar topic in the field of photovoltaic devices, where the authors investigate the photovoltaic properties of a FTO(anode)/PEDOT:PSS/FT82/C $60 / \mathrm{Al}$ (cathode) bilayer device by changing the film thickness of the polymer FT82 at the heterojunction with fullerene $\mathrm{C}_{60}$. Here, FTO and FT82 denote fluorine tin oxide and poly(9,9-dioctylfluorenealt-bithiophene), respectively. The current-voltage characteristics of this bilayer cell follows the Mott-Gurney law of space charge limited current, suggesting that the charge transport property of the bilayer photovoltaic device is of the polymer/ $\mathrm{C}_{60}$ interface.

There are five papers in this special issue dealing with a research area of fullerene nanowhiskers, metal-doped fullerene nanowhiskers, and single-crystal fullerene nanotubes. Two of the papers by T. Kizuka et al. investigate, by in situ transmission electron microscopy and measurements 
of the loading forces with an optical deflection technique, the Young's modulus of single-crystal fullerene nanotubes and nanowhiskers composed of $\mathrm{C}_{70}$ molecules. The other two papers by T. Kizuka et al. are on the synthesis and characterisation of $\mathrm{Fe}$ or $\mathrm{Ni}$-doped $\mathrm{C}_{60}$ nanowhiskers using a liquid-liquid interfacial precipitation method. Interestingly, the nanowhiskers have been observed to be able to transform into metal-encapsulated carbon nanocapsules or carbon nanotubes by heat treatments.

The paper by R. Kato and K. Miyazawa describes a photopolymerization phenomenon of $\mathrm{C}_{60}$ nanowhiskers. The authors report that the nanowhiskers can be polymerised by a laser beam, associated with a Raman spectrometer, at an appropriately high laser energy dose. The polymerized material is expected to exhibit a higher mechanical strength and a better thermal stability compared with its pristine state, a topic which deserves further studies in the future. The final paper by J. S. Soares and A. Jorio addresses how carbon nanotube properties can be affected by the surrounding environment from both a theoretical and experimental perspective. The changes in parameters such as the optical transition energy and the resonance frequency of a nanotube as a consequence of its interaction with the substrate are analyzed in this work. In addition, the transitions between metal and semiconducting properties of single-walled carbon nanotubes are explained by the tubesubstrate bonding effect.

Junfeng Geng Kun'ichi Miyazawa Zheng $\mathrm{Hu}$

Ilia A. Solov'yov

Angel Berenguer-Murcia 

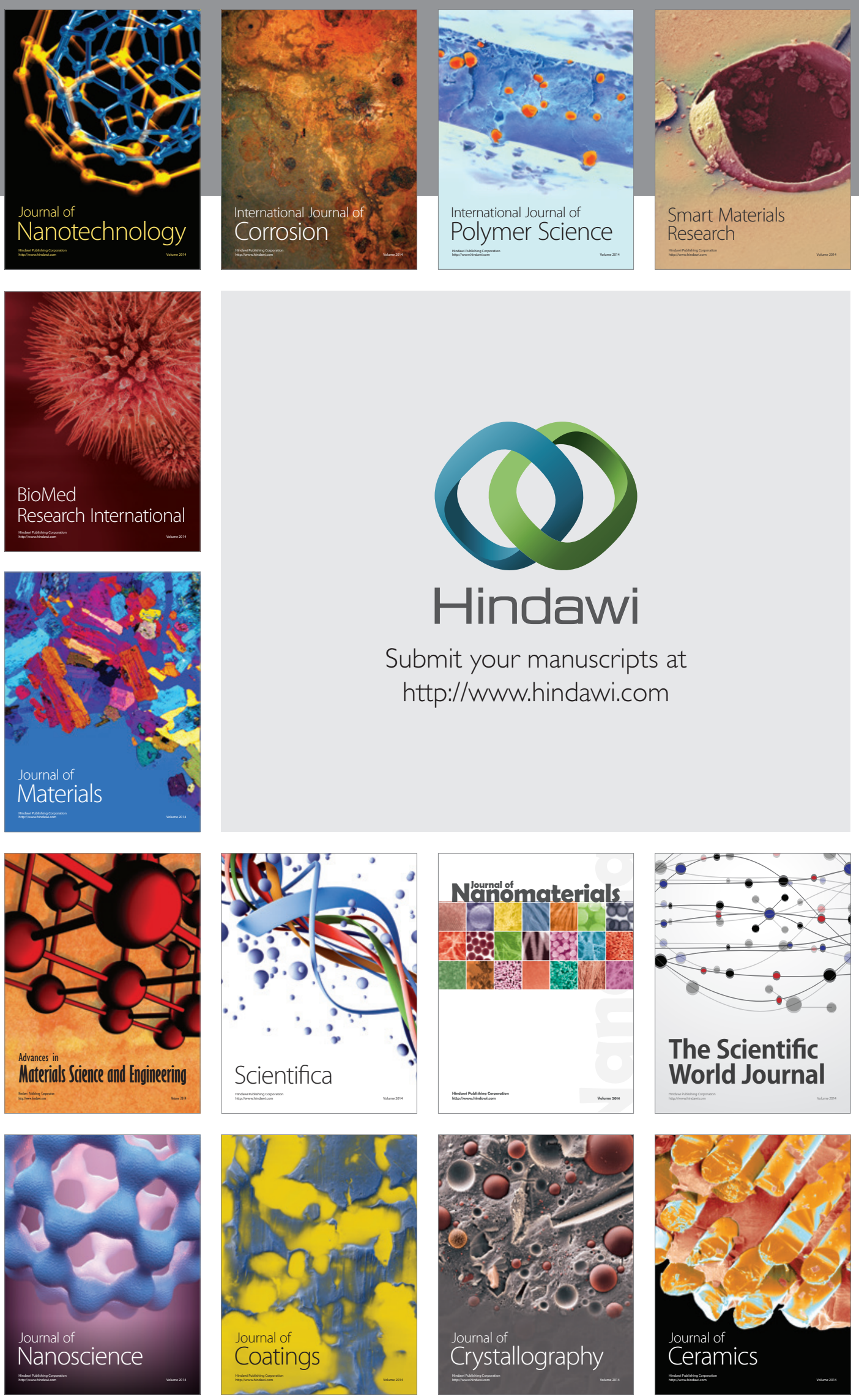

The Scientific World Journal

Submit your manuscripts at

http://www.hindawi.com

\section{World Journal}

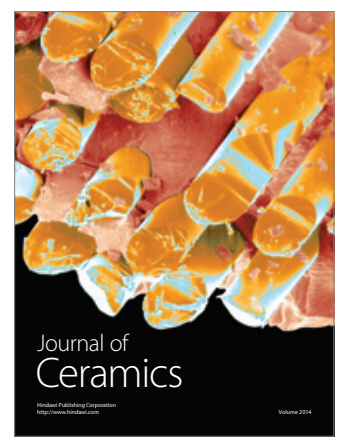

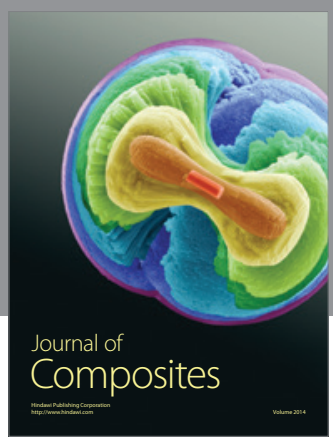
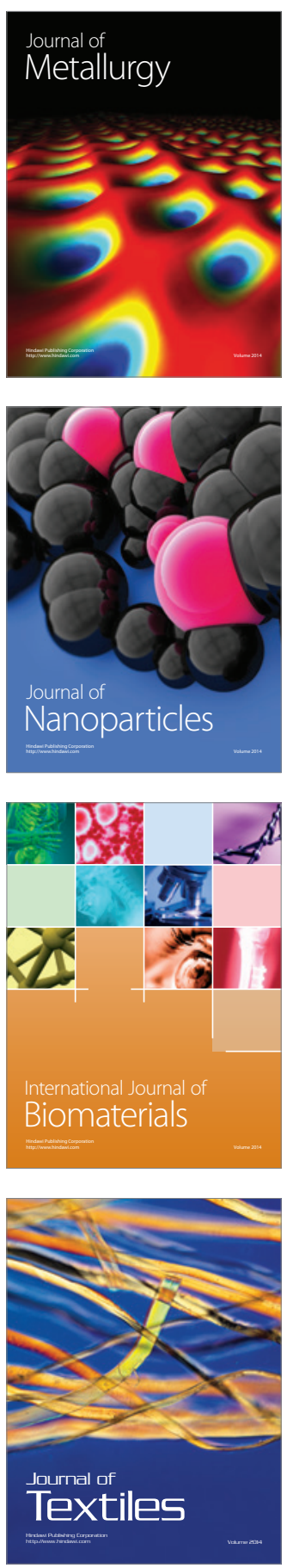